\title{
Higher Order Iteration Schemes for Unconstrained Optimization
}

\author{
Yangyang Shi ${ }^{1}$, Pingqi Pan ${ }^{2}$ \\ ${ }^{1}$ Faculty of Electrical Engineering, Mathematics and Computer Science, Delft University of Technology, \\ Delft, The Netherlands \\ ${ }^{2}$ Department of Mathematics, Southeast University, Nanjing, China \\ E-mail: shiyang1983@gmail.com,panpq@seu.edu.cn \\ Received August 3, 2011; revised August 20, 2011; accepted September 19, 2011
}

\begin{abstract}
Using a predictor-corrector tactic, this paper derives new iteration schemes for unconstrained optimization. It yields a point (predictor) by some line search from the current point; then with the two points it constructs a quadratic interpolation curve to approximate some ODE trajectory; it finally determines a new point (corrector) by searching along the quadratic curve. In particular, this paper gives a global convergence analysis for schemes associated with the quasi-Newton updates. In our computational experiments, the new schemes using DFP and BFGS updates outperformed their conventional counterparts on a set of standard test problems.
\end{abstract}

Keywords: Unconstrained Optimization, Iteration Scheme, ODE Method, Quasi-Newton Update, Convergence Analysis

\section{Introduction}

Consider the unconstrained optimization problem

$$
\min f(x) x \in R^{n}
$$

where $f: R^{n} \rightarrow R$ is twice continuously differentiable.

Let $x_{k}$ be the $k$-th iteration point. We will denote values of $f(x)$ and its gradient at $x_{k}$ by $f_{k}$ and $\nabla f_{k}$, respectively.

Optimization problems are usually solved by iteration methods. The line search widely used in unconstrained optimization is a kind of iteration scheme for updating iterates. Such a scheme, by which one obtains the next iterate $x_{k+1}$ from a current iterate $x_{k}$, is of the following form:

$$
x_{k+1}=x_{k}+\alpha p_{k} \text {, }
$$

where $p_{k}$ and $\alpha$ are termed search direction and stepsize, respectively. $p_{k}$ is usually determined as a descent direction with respect to the objective $f(x)$, and $\alpha$ by exact or inexact line searches, so that the objective value decreases after the iteration.

For instance, the famous Newton method uses the scheme with search direction

$$
p_{k}=-\left(\nabla^{2} f_{k}\right)^{-1} \nabla f_{k},
$$

where $\nabla^{2} f_{k}$ is the Hessian matrix of $f(x)$ at $x_{k}$, and stepsize $\alpha=1$.

The quasi-Newton methods are reliable and efficient in solving the unconstrained optimization problems. Saving explicit calculations of the second order derivatives and solution of a system of linear equations, quasi-Newton methods achieved a great degree of popularity since the first paper of Davidon [1,2]. He used

$$
p_{k}=-H_{k} \nabla f_{k},
$$

where $H_{k}$ is some approximation to the inverse Hessian matrix $\left(\nabla^{2} f_{k}\right)^{-1}$.

The next approximate inverse Hessian matrix $H_{k+1}$, is obtained by updating $H_{k}$ by rank-one or rank-two matrix. To this end, all quasi-Newton updates require $H_{k+1}$ for satisfying the so-called quasi-Newton equation:

$$
H_{k+1} y_{k}=s_{k} \text {, }
$$

where $y_{k}=\nabla f_{k+1}-\nabla f_{k}$ and $s_{k}=x_{k+1}-x_{k}$.

Various quasi-Newton updates were proposed in the past. The important modification of Davidon's work by Fletcher and Powell [3] (the DFP algorithm) was the first and successful one. It was then surpassed by the BFGS update (as accepted as the best quasi-Newton method) [4-8] proposed independently by Broyden, Fletcher, Goldfarb and Shanno. These updates theoretically guarantee all $H_{k}$ to be positive definite; therefore, the asso- 
ciated $p_{k}$ is a descent direction, and the objective decreases if $\alpha$ is determined by some line search.

There are other iteration schemes that appear differently from the conventional ones. The so-called ODE methods use the following initial value problem:

$$
\left\{\begin{array}{l}
\frac{\mathrm{d} x}{\mathrm{~d} t}=p(x) \\
\mathrm{d}(0)=x_{0}
\end{array}\right.
$$

Assume that $p(x)$ satisfies certain conditions, and hence the preceding defines a trajectory.

Arrow, Huwicz and Uzawa [9] used $p(x)=-\nabla f(x)$ and $p(x)=-\left(\nabla^{2} f(x)\right)^{-1} \nabla f(x)$. The associated trajectories might be called steepest descent curve and Newton curve respectively [10]. In this way, in fact, one could obtain many curves corresponding to existing unconstrained optimization methods.

Pan [11-13] generalized the steepest descent curve and Newton curve by setting $p(x)=-\phi(x) A(x)$, where $\phi(x)$ is called ratio factor and $A(x)$ direction matrix. He suggested some concrete ratio factors and direction matrices, and showed that under certain conditions, the objective value decreases strictly along the associated trajectory, the limit point of which is just an optimum.

ODE methods treat the optimization problem in the view of trajectory. They use numerical methods to approximately calculate associated trajectory, and finally approach the limit point of the trajectory. When Euler's approach is applied in the ODE method, standard iteration schemes are obtained. In fact the standard iteration schemes are originally derived in the direction of decreasing the objective function value instead of trajectory. Euler's approach is only of the first order precision. So it is possible to apply higher order approach to mimic the trajectory to get higher order iteration scheme than the standard one.

In this paper, we derive new iteration schemes along this line. In view of the importance of DFP and BFGS methods, we will focus on iteration schemes with respect to these methods.

The paper is organized as follows. Section 2 derives new iteration schemes. Section 3 offers the convergence analysis. Section 4 reports encouraging computational results with a set of problems.

\section{Higher Order Iteration Scheme}

Assume that $x_{k}$ is the current iterate. The next iterate $x_{k+1}$ will be determined by approximately following the trajectory, defined by (4). Let $\tilde{x}_{k+1}$ be a predictor. Introduce notation

$$
p_{k}=p_{k}\left(x_{k}\right), \tilde{p}_{k+1}=p\left(\tilde{x}_{k+1}\right) .
$$

We construct a quadratic interpolation curve, locally approximating the trajectory as follows:

$$
x(t)=a_{k} t^{2}+b_{k} t+c_{k},
$$

where $a_{k}, b_{k}, c_{k}$ satisfy the following conditions:

$$
\begin{gathered}
a_{k} t_{k}^{2}+b_{k} t_{k}+c_{k}=x_{k}, \\
2 a_{k} t_{k}+b_{k}=p_{k}, \\
a_{k} \tilde{t}_{k+1}^{2}+b_{k} \tilde{t}_{k+1}+c_{k}=\tilde{x}_{k+1}, \\
2 a_{k} \tilde{t}_{k+1}+b_{k}=\tilde{p}_{k+1} .
\end{gathered}
$$

Set $\tilde{t}_{k+1}=0$, so $x(0)=\tilde{x}_{k+1}$. From (6a)-(6d), it is easily to draw that $b_{k}=\tilde{p}_{k+1}, \quad c_{k}=\tilde{x}_{k+1}$ and

$$
\begin{gathered}
\frac{\tilde{p}_{k+1}+p_{k}}{2} t_{k}=x_{k}-\tilde{x}_{k+1}, \\
a_{k} t_{k}=\frac{p_{k}-\tilde{p}_{k+1}}{2} .
\end{gathered}
$$

Pre-multiplying the both sides of (7a) by $\left(x_{k}-\tilde{x}_{k+1}\right)^{T}$, we obtain an approximate $t_{k-1}$, furthermore, have an approximate solution of (6a)-(6d).

$$
\begin{gathered}
a_{k}=\frac{\left(p_{k}-\tilde{p}_{k+1}\right)\left(x_{k}-\tilde{x}_{k+1}\right)^{T}\left(p_{k}+\tilde{p}_{k+1}\right)}{4\left\|\left(x_{k}-\tilde{x}_{k+1}\right)\right\|^{2}}, \\
b_{k}=\tilde{p}_{k+1}, \quad c_{k}=\tilde{x}_{k+1},
\end{gathered}
$$

where $\left\|\left(x_{k}-\tilde{x}_{k+1}\right)\right\|$ denotes 2-norm of the vector $\left(x_{k}-\tilde{x}_{k+1}\right)$.

The unconstrained optimization problem (1) can get a approximate solution by solving the following one-dimension minimization problem:

$$
\min \varphi(t)=f\left(a_{k} t^{2}+b_{k} t+c_{k}\right), t \geq 0 .
$$

To solve such problem, we apply the inexact line search rule, furthermore, we modify the sufficient decent condition

$$
f\left(x_{k}+t p_{k}\right) \leq f\left(x_{k}\right)+\rho t p_{k}^{T} \nabla f_{k},
$$

in this way:

$$
f\left(a_{k} t^{2}+b_{k} t+c_{k}\right) \leq f\left(c_{k}\right)+\rho t b_{k}^{T} \nabla f\left(c_{k}\right),
$$

where $\rho \in(0,1)$.

\subsection{Modified Inexact Line Search Algorithm}

The conventional backtracking inexact line search [14] operates in this way. At the beginning we set $t=\hat{t}$. The algorithm will stop if $t$ satisfies the sufficient decent condition. Otherwise, the algorithm will continue with $t \leftarrow \alpha t$.

A modified backtracking inexact line search algorithm 
was obtained by applying the expression (12) as the sufficient decent condition in backtracking line search algorithm.

Subalgorithm 2.1 modified backtracking inexact line search

Step 0. Given $\hat{t}, \rho, \alpha \in(0,1), \varepsilon$; set $t=\hat{t}$.

Step 1. Set $a_{k}=0$ if $a_{k}^{T} \nabla f_{k}>-c b_{k}^{T} \nabla f_{k}$, where constant $c \geq \frac{1}{\hat{t}}$.

Step 2. If

$$
f\left(a_{k} t^{2}+b_{k} t+c_{k}\right) \leq f\left(c_{k}\right)+\rho t b_{k}^{T} \nabla f\left(c_{k}\right),
$$

go to step 4; otherwise, go to step 3 .

Step 3. $t \leftarrow \alpha t$ and go to step 2 .

Step 4. Terminate with $t_{k}=t$.

\subsection{Higher Order Iteration Schemes}

The higher order iteration schemes, firstly obtain the predictor $\tilde{x}_{k+1}$ from the current point $x_{k}$ by inexact line search rule following the direction $p_{k}$. Then construct the quadratic interpolation curve by the relevant information of $x_{k}$ and $\tilde{x}_{k+1}$, and calculate $x_{k+1}$ satisfying modified inexact line search rule (12). The overall steps of the higher order iteration schemes are organized as follows.

\section{Algorithm 2.1 Higher order iteration schemes}

Step 0. Given initial point $x_{0}, \rho, \alpha \in(0,1)$ and $\varepsilon$, set $k:=0$;

Step 1. If $\left\|\nabla f_{k}\right\|<\varepsilon$, stop.

Step 2. compute the predictor

- Call backtracking inexact line search algorithm to obtain $t_{k}$.

- Compute $\tilde{x}_{k+1}=x_{k}+t_{k} p_{k}$.

Step 3. If $\left\|\nabla f\left(\tilde{x}_{k+1}\right)\right\|<\varepsilon$, stop.

Step 4. Compute a new iteration point.

- Compute $a_{k}, b_{k}, c_{k}$ by (8) and (9).

- Call subalgorithm 2.1 to get $\tilde{t}_{k+1}$.

- Compute $x_{k+1}=a_{k} \tilde{t}_{k+1}^{2}+b_{k} \tilde{t}_{k+1}+c_{k}$.

Step 5. Set $k:=k+1$ and go to step 1 .

\subsection{An Extension of the Higher Order Iteration Scheme}

The higher order iteration schemes vary with different $p(x)$. In this paper we extend the higher order iteration schemes to BFGS method, and set $p_{k}=-H_{k} \nabla f_{k}$. We get the predictor $\tilde{X}_{k+1}$ from $x_{k}$, satisfying the backtracking inexact line search rule, in the direction $p_{k}$. From $x_{k}$ and $\tilde{x}_{k+1}$, we compute $\tilde{p}_{k+1}=-\tilde{H}_{k+1} \nabla \tilde{f}_{k+1}$. Then by searching along the curve, we obtain the new point $x_{k+1}$. The overall steps of the variant of the itera- tion schemes are organized as follows.

Algorithm 2.2 higher order iteration schemes using BFGS update

Step 0. Given initial point $x_{0}$; set $k:=0, \rho, \alpha \in(0,1)$, $N, H_{0}=I, \varepsilon$ and $\varepsilon$;

Step 1. Compute the predictor.

- Call backtracking inexact line search algorithm to obtain $t_{k}$.

- Compute $\tilde{x}_{k+1}=x_{k}-t_{k} H_{k} \nabla f_{k}$ and $\tilde{f}_{k+1}=f\left(\tilde{x}_{k+1}\right)$.

Step 2. If $\left\|\nabla f\left(\tilde{x}_{k+1}\right)\right\|<\varepsilon$, stop.

Step 3. Compute $\tilde{H}_{k+1}$.

- If $\tilde{y}_{k}^{T} \tilde{s}_{k} \leq \varepsilon$ then $\tilde{H}_{k+1}=I$; otherwise

$$
\tilde{H}_{k+1}=H_{k}+\left(1+\frac{\tilde{y}_{k}^{T} H_{k} \tilde{y}_{k}}{\tilde{s}_{k}^{T} \tilde{y}_{k}}\right) \frac{\tilde{s}_{k} \tilde{s}_{k}^{T}}{\tilde{s}_{k}^{T} \tilde{y}_{k}}-\frac{\tilde{s}_{k} \tilde{y}_{k}^{T} H_{k}+H_{k} \tilde{y}_{k} \tilde{s}_{k}^{T}}{\tilde{s}_{k}^{T} \tilde{y}_{k}},
$$

where $\tilde{y}_{k}=\nabla \tilde{f}_{k+1}-\nabla f_{k}, \quad \tilde{s}_{k}=-t_{k} H_{k} \nabla f_{k}$.

Step 4. Compute the new iteration point.

- Compute $a_{k}, b_{k}, c_{k}$ by (8) and (9).

- Call subalgorithm 2.1 to get $\tilde{t}_{k+1}$.

- Compute $x_{k+1}=a_{k} \tilde{t}_{k+1}^{2}+b_{k} \tilde{t}_{k+1}+c_{k}$.

Step 5. If $\left\|\nabla f_{k+1}\right\|<\varepsilon$, stop.

Step 6. Update $H_{k}$.

- If $k+1(\bmod N)=0$ or $y_{k}^{T} s_{k} \leq \varepsilon$ then $H_{k+1}=I$; otherwise

$$
H_{k+1}=H_{k}+\left(1+\frac{y_{k}^{T} H_{k} y_{k}}{s_{k}^{T} y_{k}}\right) \frac{s_{k} s_{k}^{T}}{s_{k}^{T} y_{k}}-\frac{s_{k} y_{k}^{T} H_{k}+H_{k} y_{k} s_{k}^{T}}{s_{k}^{T} y_{k}},
$$

where $y_{k}=\nabla f_{k+1}-\nabla f_{k}, \quad s_{k}=x_{k+1}-x_{k}$

- If $\left(\frac{\nabla f_{k+1}^{T} H_{k+1} \nabla f_{k+1}}{\left\|\nabla f_{k+1}\right\|\left\|H_{k+1} \nabla f_{k+1}\right\|}\right)<\varepsilon$ or $\frac{\left\|\nabla f_{k+1}^{T} H_{k+1}\right\|}{\left\|\nabla f_{k+1}\right\|}<\varepsilon$, then set $H_{k+1}=I$.

Step 7. Set $k:=k+1$ and go to step 1 .

Note:

1) In this paper, I denotes identity matrix.

2) In step 3, we adopt a strategy to make sure that the curvature condition $\tilde{y}_{k}^{T} \tilde{s}_{k}>\varepsilon$ is hold. So $\tilde{H}_{k+1}$ is positive and $\tilde{F}_{k+1}$ is a decent direction.

3) In step 4, we call the subalgorithm (2.1), in which we set the parameter $c=\frac{1}{\varepsilon}$.

4) In step 6, we adopt a restart technique that if $k+1$ is the integral multiple of the $N$ or $y_{k}^{T} s_{k} \leq \varepsilon$, we restart with $H_{k+1}=I$. Clearly, the BFGS method is a kind of conjugate direction method, so the restart technique can reduce the accumulation of the roundoff errors.

5) We only report the variant using BFGS update. we also derive a variant of the higher order iteration schemes by using the DFP updating formula instead of the BFGS 
updating formula in step 3 and step 6.

\section{The Global Convergence of the Higher Order Iteration Schemes}

Definition 3.1 [11] Curve $x(t)$ where $t \in(0, \beta)$, if for any $\beta>0, t \in(0, \beta), x(t)$ is contained in the domain of $f(x)$ and $f(x(t))$ is strictly monotone decreasing in $(0, \beta)$, then $x(t)$ is a decent trajectory of $f(x)$ at $x(0)=x_{0}$. And if $\lim _{t \rightarrow \beta_{-}} x(t)$ is exist and equal the minimization point of $f(x)$, then the curve $x(t)$ is normal decent trajectory of $f(x)$ at $x_{0}$.

Pan proved the global convergence of the ODE methods with ratio factor and direction matrix [11]. In this paper we only consider the situation that ratio factor is 1 and direction matrix is identity matrix. So we draw the theorem as follows,

Theorem 3.1 [11] Given $x_{0} \in \mathbb{R}^{n}$, assume the level set

$$
\mathcal{L}=\left\{x: x \in \mathbb{R}^{n}, f(x) \leq f\left(x_{0}\right)\right\}
$$

is bounded close set, and $f(x)$ is twice continuously differentiable in the set $\mathcal{L}$ and $\nabla f\left(x_{0}\right) \neq 0$, then the right segment trajectory of the the ordinary equations (4) is the decent curve of $f(x)$ at $x_{0}$ and the limit point of the trajectory is the stationery point of $f(x)$. If $f(x)$ is convex, then the right segment trajectory is normal decent curve of $f(x)$ at $x_{0}$.

We use the quadratic interpolation curve (5) to approximate the trajectory. However, when $a_{k}^{T} \nabla f_{k}>0$, the iteration scheme may not be decent in the local region of the predictor $\tilde{x}_{k+1}$. So we apply the strategy of step 1 in subalgorithm (2.1) to keep the iteration decent.

Theorem 3.2 Given constant $\hat{t}$ and $c \geq \frac{1}{\hat{t}}$. In subalgorithm (2.1), if $b_{k}$ is decent direction satisfying

$$
b_{k}^{T} \nabla f_{k} \leq 0,
$$

then for any $0<t \leq \frac{1}{c}$, the condition

$$
\left(a_{k} t^{2}+b_{k} t\right)^{T} \nabla f_{k} \leq 0
$$

is hold.

Proof. If $a_{k}^{T} \nabla f_{k} \leq 0$, clearly, the conclusion holds.

Otherwise $a_{k}^{T} \nabla f_{k}>0$, from algorithm (2.1) step 1, if

$$
a_{k}^{T} \nabla f_{k}>-c b_{k}^{T} \nabla f_{k},
$$

then set $a_{k}=0$, so the conclusion holds. If

$$
a_{k}^{T} \nabla f_{k} \leq-c b_{k}^{T} \nabla f_{k},
$$

then

$$
\left(a_{k} t^{2}+b_{k} t\right)^{T} \nabla f_{k} \leq t\left(\frac{1}{c} a_{k}+b_{k}\right)^{T} \nabla f_{k} \leq 0 .
$$

Theorem 3.3 Consider the algorithm (2.1), where $p_{k}$ and $\tilde{p}_{k}$ are decent direction, and $p_{k}$ satisfying the condition

$$
\frac{-p_{k}^{T} \nabla f_{k}}{\left\|p_{k}\right\|\left\|\nabla f_{k}\right\|} \geq c_{1}
$$

and

$$
\left\|p_{k}\right\| \geq c_{2}\left\|\nabla f_{k}\right\|
$$

where $c_{1}>0$ and $c_{2}>0$ are constants. Suppose that $f(x)$ is bounded below and continuously differentiable in the level set

$$
\mathcal{L}=\left\{x: x \in \mathbb{R}^{n}, f(x) \leq f\left(x_{0}\right)\right\},
$$

where $x_{0}$ is the starting point. And the gradient $\nabla f$ is Lipschitz continuous on $\mathcal{L}$; namely, there exists $L$ such that

$$
\|\nabla f(x)-\nabla f(y)\| \leq L\|x-y\| .
$$

Then for some $k, \nabla f_{k}=0$ is hold, otherwise,

$$
\lim _{k \rightarrow+\infty}\left\|\nabla f_{k}\right\|=0 \text {. }
$$

Proof. Consider the situation that for all $k, \nabla f_{k} \neq 0$. Then from the algorithm (2.1), we have that

$$
\tilde{f}_{k+1} \leq f_{k}+\rho t_{k} p_{k}^{T} \nabla f_{k},
$$

and

$$
f_{k+1} \leq \tilde{f}_{k+1}+\rho \tilde{t}_{k+1} \tilde{p}_{k+1}^{T} \nabla \tilde{f}_{k+1} \leq \tilde{f}_{k+1},
$$

By (21) and (22), we have

$$
f_{k}-\tilde{f}_{k+1} \geq-\rho t_{k} p_{k}^{T} \nabla f_{k},
$$

and

$$
\tilde{f}_{k+1}-f_{k+1} \geq 0 .
$$

With (23) and (24), we obtain

$$
f_{k}-f_{k+1} \geq-\rho t_{k} p_{k}^{T} \nabla f_{k} .
$$

By summing this expression over all indices less than or equal to $k$, we obtain

$$
f_{0}-f_{k+1} \geq-\rho \sum_{j=0}^{k} t_{k} p_{k}^{T} \nabla f_{k} .
$$

Since $f$ is bounded below, we have that $f_{0}-f_{k+1}$ is less than some positive constant, for all $k$. Hence, by taking limits in (26), we obtain 


$$
\sum_{j=0}^{\infty}-t_{k} p_{k}^{T} \nabla f_{k}<+\infty .
$$

In standard inexact line search algorithem, we know that if the initiate trial $\hat{t}$ does not satisfy the condition (11), then $\frac{t_{k}}{\alpha}$ violate the condition. So

$$
f\left(x_{k}+\frac{t_{k}}{\alpha} p_{k}\right)>f_{k}+\rho \frac{t_{k}}{\alpha} p_{k}^{T} \nabla f_{k} .
$$

By the Lipschitz condition (37), we have

$$
\begin{aligned}
& \tilde{f}_{k+1}-f_{k}=f\left(x_{k}+t_{k} p_{k}\right)-f_{k} \\
& =t_{k} p_{k}^{T} \nabla f_{k}+\int_{0}^{t_{k}}\left[\nabla f\left(x_{k}+s p_{k}\right)-\nabla f_{k}\right]^{T} p_{k} \mathrm{~d} s \\
& \leq t_{k} p_{k}^{T} \nabla f_{k}+\int_{0}^{t_{k}} s L\left\|p_{k}\right\|^{2} \mathrm{~d} s \\
& =t_{k} p_{k}^{T} \nabla f_{k}+\left(\frac{1}{2}\right) L t_{k}^{2}\left\|p_{k}\right\|^{2} \\
& \leq \rho t_{k} p_{k}^{T} \nabla f_{k}, \text { for all } 0<t_{k} \leq \frac{2(\rho-1) p_{k}^{T} \nabla f_{k}}{L\left\|p_{k}\right\|^{2}}
\end{aligned}
$$

It follows from (28) and (29) that

$$
\frac{t_{k}}{\alpha}>\frac{2(\rho-1) p_{k}^{T} \nabla f_{k}}{L\left\|p_{k}\right\|^{2}} .
$$

If initiate trial $\hat{t}$ satisfy the condition (11), then $t=\hat{t}$. Furthermore, from (16) and (17), we have

$$
t_{k} \geq \min \left\{\frac{2 \alpha(\rho-1) p_{k}^{T} \nabla f_{k}}{L\left\|p_{k}\right\|^{2}}, \hat{t}\right\}>0 .
$$

It follows from (27) that

$$
\sum_{j=0}^{\infty} \min \left\{\frac{2 \alpha(\rho-1)\left|p_{k}^{T} \nabla f_{k}\right|^{2}}{L\left\|p_{k}\right\|^{2}},-\hat{t} p_{k}^{T} \nabla f_{k}\right\}<+\infty
$$

It follows from (16) and (17)

$$
\sum_{j=0}^{\infty} \min \left\{\frac{2 \alpha(1-\rho)}{L} c_{1}^{2}\left\|p_{k}\right\|^{2}, \hat{t} c_{1} c_{2}\left\|\nabla f_{k}\right\|^{2}\right\}<+\infty
$$

By (33), we obtain that

$$
\sum_{j=0}^{\infty}\left\|\nabla f_{k}\right\|^{2}<+\infty
$$

This implies that

$$
\lim _{k \rightarrow+\infty}\left\|\nabla f_{k}\right\|=0 .
$$

The theorem (3.3) analyzes the global convergence of the iteration scheme based on ODE, similarly, we obtain the global convergence of the variant iteration scheme using BFGS update formula.
Theorem 3.4 Consider the algorithm (2.2), suppose that $f$ is bounded below in $\mathbb{R}^{n}$ and continuously differentiable in the level set

$$
\mathcal{L}=\left\{x \mid f(x) \leq f\left(x_{0}\right)\right\},
$$

where $x_{0}$ is the starting point. And the gradient $\nabla f$ is Lipschitz continuous on $\mathcal{L}$; namely, there exists $L$ such that

$$
\|\nabla f(x)-\nabla f(y)\| \leq L\|x-y\| .
$$

Then for some $k, \nabla f_{k}=0$ is hold, otherwise,

$$
\lim _{k \rightarrow+\infty}\left\|\nabla f_{k}\right\|=0 \text {. }
$$

Proof. Consider the situation that for all $k, \nabla f_{k} \neq 0$. Then from the algorithm (2.2), we have that

$$
f_{k+1} \leq \tilde{f}_{k+1}-\rho \tilde{t}\left(\tilde{H}_{k+1} \nabla \tilde{f}_{k+1}\right)^{T} \nabla \tilde{f}_{k+1}
$$

and

$$
\tilde{f}_{k+1} \leq f_{k}-\rho t\left(H_{k} \nabla f_{k}\right)^{T} \nabla f_{k} .
$$

The step 3 implies that

$$
\left(\tilde{H}_{k+1} \nabla \tilde{f}_{k+1}\right)^{T} \nabla \tilde{f}_{k+1}>0
$$

By combining the condition (39), (40) and (41), we have

$$
f_{k}-f_{k+1} \geq \rho t\left(H_{k} \nabla f_{k}\right)^{T} \nabla f_{k} .
$$

From the step 8 in the algorithm (2.2), we have that

$$
\frac{\nabla f_{k+1}^{T} H_{k+1} \nabla f_{k+1}}{\left\|\nabla f_{k+1}\right\|\left\|H_{k+1} \nabla f_{k+1}\right\|} \geq \varepsilon
$$

and

$$
\frac{\left\|\nabla f_{k+1}^{T} H_{k+1}\right\|}{\left\|\nabla f_{k+1}\right\|} \geq \varepsilon .
$$

From the theorem (3.3), we conclude that

$$
\lim _{k \rightarrow+\infty}\left\|\nabla f_{k}\right\|=0 \text {. }
$$

is hold.

\section{Computational Results}

In this section, we report computational results showing that the variant iteration schemes using BFGS and DFP update formula outperformed the BFGS method and DFP method on two sets of test functions. The first set of 20 functions were from [15], and the second from [16], which can be obtained from http://www.ici.ro/camo/neculai/ ansoft.htm. 


\subsection{Test Codes}

In this section, the following four codes are tested:

- DFP: the DFP method.

- BFGS: the BFGS method.

- HDFP: the higher order iteration schemes using DFP update.

- HBFGS: the higher order iteration schemes using BFGS update.

To have the competitions fair and easy, all the codes were implemented with the same parameters: $\rho=10^{-4}$, $\alpha=0.5, \quad N=15, H_{0}=I, \varepsilon=10^{-6}, \varepsilon=10^{-12}$ and $\hat{t}=1$; The detail results of the BFGS and HBFGS are showed below. And the performance of DFP and HDFP are only demonstrated in the overall results table.

Compiled using Matlab 7.0.4, the four codes operated under a Windows XP system Home Edition Version 2002 on an Asus PC with Genuine Intel(R) CentrinoDuo T2300 processor $1.66 \mathrm{GHz}$ and 1.00 GB memory.

\subsection{Result for 20 Small Scale Functions}

The first set of test problems included the 20 problems. Numerical results obtained are listed in Table 1, where numbers of function value computation and gradient computation are listed in columns labeled " $f$ " and " $\nabla f$ ", respectively. And CUP-time required for solving each problem are listed in columns labeled 'Time' and its unit is second. "_" denotes that the algorithm does not get a correct solution in upper bound iteration number.

Table 1 serves as an comparison between the BFGS and HBFGS. It shows that the computation numbers of function value andgradient vectors of HBFGS are fewer than that of BFGS. However, the HBFGS costs 0.11 seconds more than the BFGS, because the HBFGS has to compute $a_{k}$. Although the computation of $a_{k}$ is much less compare with that of function value, it affects the CPU-time, especially, for small scale problems. So the HBFGS is competitive with BFGS on the 20 small scale

Table 1. Statistics of first 20 functions.

\begin{tabular}{|c|c|c|c|c|c|c|}
\hline \multirow{2}{*}{ Problem } & \multicolumn{3}{|c|}{ BFGS } & \multicolumn{3}{|c|}{ HBFGS } \\
\hline & Time & $f$ & $\nabla f$ & Time & $f$ & $\nabla f$ \\
\hline Rosenbrock & 0.02 & 62 & 36 & 0.06 & 133 & 44 \\
\hline Freudenstein and Roth & 0.00 & 29 & 14 & 0.03 & 48 & 16 \\
\hline Powell badly scaled & 0.17 & 796 & 303 & 0.02 & 257 & 33 \\
\hline Brown badly scaled & - & - & - & - & - & - \\
\hline Beale & 0.03 & 23 & 16 & 0.03 & 35 & 18 \\
\hline Jennrich and Sampson & 0.00 & 7 & 2 & 0.02 & 7 & 2 \\
\hline Helical vally & 0.05 & 77 & 35 & 0.06 & 166 & 44 \\
\hline Bard & 0.05 & 430 & 34 & 0.03 & 65 & 22 \\
\hline Guassian & 0.00 & 6 & 5 & 0.02 & 20 & 10 \\
\hline Meyer & - & - & - & - & - & - \\
\hline Gulf research and develop & 0.05 & 1 & 2 & 0.00 & 1 & 2 \\
\hline Box three-dimensional & 0.04 & 51 & 41 & 0.05 & 86 & 44 \\
\hline Powell singular & 0.05 & 65 & 36 & 0.06 & 157 & 58 \\
\hline Wood & 0.05 & 88 & 32 & 0.06 & 194 & 44 \\
\hline Kowalik and Osborne & 0.05 & 45 & 42 & 0.05 & 71 & 38 \\
\hline Brown and Dennis & 0.34 & 2546 & 263 & 0.20 & 1225 & 104 \\
\hline Biggs EXP6 & 0.03 & 21 & 19 & 0.05 & 31 & 20 \\
\hline Watson & 0.39 & 79 & 42 & 0.47 & 163 & 54 \\
\hline Extended Rosenbrock & 0.13 & 244 & 74 & 0.25 & 832 & 130 \\
\hline Broyden banded & 0.09 & 160 & 45 & 0.19 & 647 & 112 \\
\hline total & 1.53 & 4730 & 1041 & 1.64 & 4138 & 795 \\
\hline
\end{tabular}


problems.

\subsection{Result for 50 Middle Scale Functions}

The second test set of 50 problems consist of 43 functions with 100 variables, 3 functions with 200 variables and 4 functions with 300 variables. The problems with “*” have 300 independent variables, and with “**” have 200 independent variables.

Table 2 shows that compared with BFGS, the computation of the function value and the gradient vectors and CPU-time of HBFGS decrease by 52.65\%, 52.08\% and $36.01 \%$, respectively. In summary the HBFGS method are faster and have less computation than the BFGS method.

\subsection{Result for 50 Large Scale Functions}

The second test set of 50 problems consist of 46 functions with 500 variables, 4 functions with 300 variables. The problems with “*” have 300 independent variables.

Table 3 shows that the HBFGS's CPU-time, computation numbers of function value and gradient vectors are less than the BFGS by 949.10 seconds, 38808 and 3957, respectively.

\subsection{Statistics of the Ratio}

The Table 4 gives overall comparison of HDFP, HBFGS and DFP, BFGS. In Table 4, "Time" denotes the run time ratio, " $f$ " denotes function value computation number ratio and " $\nabla f$ " denotes gradient computation number ratio.

Table 4 shows that the HDFP outperforms the DFP with the average CPU-time ratio 1.58, function computation ratio 1.67 and gradient computation ratio 1.71. And the HBFGS defeats the BFGS with the average CPUtime ratio 1.23 , function computation ratio 1.57 and gradient computation ratio 1.56 .

\subsection{Summary of the Tests}

As the tests show, although the higher order iteration schemes add the computation of $a_{k}$, it has less computation of function value and gradient vector. For large scale problems, the computation of $a_{k}$ is much less than that of function value.

\section{Concluding Remarks}

We gave a new iteration scheme based on ODE, proved

Table 2. Statistics of middle scale 50 functions.

\begin{tabular}{|c|c|c|c|c|c|c|}
\hline \multirow{2}{*}{ Problem } & \multicolumn{3}{|c|}{ BFGS } & \multicolumn{3}{|c|}{ HBFGS } \\
\hline & Time & $f$ & $\nabla f$ & Time & $f$ & $\nabla f$ \\
\hline Strictly Convex1 & 0.05 & 7 & 8 & 0.06 & 21 & 12 \\
\hline Strictly Convex2 & 0.30 & 202 & 97 & 0.19 & 147 & 60 \\
\hline Extended Freudenstein and Roth & 0.08 & 21 & 12 & 0.13 & 45 & 18 \\
\hline Extended Trigonometric & 0.17 & 237 & 57 & 0.39 & 388 & 124 \\
\hline Extended White and Holst & 0.19 & 75 & 36 & 0.22 & 118 & 38 \\
\hline Extended Beale & 0.08 & 20 & 15 & 0.09 & 45 & 20 \\
\hline Extended Penalty & 0.09 & 85 & 26 & 0.13 & 141 & 38 \\
\hline Perturbed Quadratic & 0.80 & 1729 & 279 & 0.53 & 1095 & 170 \\
\hline Diagonal2 & 0.28 & 112 & 113 & 0.27 & 97 & 96 \\
\hline Diagonal1 & 5.58 & 10756 & 1998 & 1.03 & 1701 & 342 \\
\hline Diagonal3 & 5.77 & 10380 & 2001 & 1.03 & 1758 & 326 \\
\hline Hager & 0.16 & 138 & 63 & 0.13 & 107 & 40 \\
\hline Generalized Tridiagonal-1 & 0.47 & 460 & 104 & 0.44 & 439 & 90 \\
\hline Extended Tridiagonal-1 & 0.19 & 30 & 28 & 0.17 & 29 & 24 \\
\hline Extended Three Exponential Terms & 0.06 & 12 & 9 & 0.05 & 33 & 12 \\
\hline
\end{tabular}




\begin{tabular}{|c|c|c|c|c|c|c|}
\hline Generalized Tridiagonal-2 & 0.67 & 1261 & 189 & 0.77 & 1340 & 192 \\
\hline Diagonal4 & 0.03 & 9 & 4 & 0.03 & 28 & 6 \\
\hline Diagonal5 & 0.03 & 5 & 6 & 0.03 & 12 & 8 \\
\hline Extended Himmelblau & 0.06 & 19 & 10 & 0.06 & 56 & 18 \\
\hline Generalized PSC1 & 0.73 & 369 & 313 & 0.28 & 137 & 104 \\
\hline Extended PSC1 & 0.06 & 22 & 15 & 0.09 & 41 & 22 \\
\hline Extended Powell** & 1.45 & 148 & 78 & 0.98 & 111 & 50 \\
\hline Extended Block Diagonal BD1 & 0.05 & 14 & 13 & 0.06 & 30 & 16 \\
\hline Extended Maratos & 0.19 & 146 & 67 & 0.25 & 264 & 90 \\
\hline Extended Cliff & 0.17 & 71 & 44 & 0.09 & 65 & 22 \\
\hline Quadratic Diagonal Perturbed & 0.11 & 57 & 40 & 0.13 & 65 & 42 \\
\hline Extended Wood** & 0.34 & 81 & 25 & 0.64 & 134 & 44 \\
\hline Scaled Quadratic SQ1** & 6.61 & 3163 & 460 & 5.70 & 3205 & 360 \\
\hline Quadratic Function QF1 & 0.52 & 1015 & 193 & 0.42 & 784 & 140 \\
\hline Extended Quadratic Penalty QP1 & 0.06 & 41 & 21 & 0.06 & 45 & 16 \\
\hline Extended Quadratic Penalty QP2 & 0.08 & 49 & 26 & 0.11 & 82 & 34 \\
\hline A Quadratic Function QF2 & 0.92 & 2352 & 324 & 0.58 & 1377 & 186 \\
\hline Extended EP1 & 0.03 & 12 & 5 & 0.05 & 31 & 8 \\
\hline Extended Tridiagonal-2 & 0.11 & 75 & 40 & 0.11 & 83 & 38 \\
\hline BDQRTIC & 1.70 & 5044 & 560 & 0.69 & 1619 & 206 \\
\hline TRIDIA & 1.75 & 5288 & 570 & 0.88 & 2336 & 258 \\
\hline ARWHEAD & 1.69 & 749 & 121 & 1.81 & 748 & 114 \\
\hline NONDIA (Shanno-78) & 0.06 & 88 & 19 & 0.19 & 378 & 58 \\
\hline NONDQUAR & 5.25 & 2170 & 1999 & 2.86 & 1207 & 1040 \\
\hline DQDRTIC & 0.05 & 49 & 13 & 0.14 & 234 & 42 \\
\hline Extended Rosenbrock & 0.13 & 74 & 38 & 0.16 & 153 & 52 \\
\hline EG2 & 0.05 & 21 & 12 & 0.08 & 57 & 24 \\
\hline DIXMAANA* & 0.48 & 14 & 11 & 0.75 & 27 & 16 \\
\hline DIXMAANB* & 0.69 & 19 & 16 & 0.94 & 31 & 20 \\
\hline Almost Perturbed Quadratic & 0.80 & 1744 & 280 & 0.47 & 963 & 148 \\
\hline Tridiagonal Perturbed Quadratic & 1.25 & 1686 & 267 & 0.83 & 1088 & 168 \\
\hline DIXMAANC** & 0.67 & 21 & 16 & 0.98 & 40 & 20 \\
\hline DIXMAANE* & 8.31 & 176 & 174 & 4.93 & 109 & 102 \\
\hline Partial Perturbed Quadratic & 0.97 & 1618 & 260 & 0.64 & 1019 & 158 \\
\hline Broyden Tridiagonal & 0.64 & 1030 & 174 & 0.66 & 1015 & 160 \\
\hline total & 50.97 & 52964 & 11251 & 32.31 & 25078 & 5392 \\
\hline
\end{tabular}


Table 3. Statistics of large scale 50 functions on BFGS and HBFGS.

\begin{tabular}{|c|c|c|c|c|c|c|}
\hline \multirow{2}{*}{ Problem } & \multicolumn{3}{|c|}{ BFGS } & \multicolumn{3}{|c|}{ HBFGS } \\
\hline & Time & $f$ & $\nabla f$ & Time & $f$ & $\nabla f$ \\
\hline Strictly Convex1 & 1.58 & 7 & 8 & 2.13 & 22 & 12 \\
\hline Strictly Convex2 & 383.11 & 8094 & 2001 & 346.83 & 6694 & 1714 \\
\hline Extended Freudenstein and Roth & 3.14 & 21 & 12 & 5.83 & 48 & 20 \\
\hline Extended Trigonometric & 12.84 & 411 & 66 & 48.61 & 706 & 242 \\
\hline Extended White and Holst & 9.36 & 75 & 36 & 11.09 & 123 & 40 \\
\hline Extended Beale & 3.39 & 20 & 15 & 4.83 & 45 & 20 \\
\hline Extended Penalty & 116.31 & 6041 & 580 & 32.44 & 309 & 17 \\
\hline Perturbed Quadratic & 81.64 & 3428 & 398 & 86.69 & 3508 & 400 \\
\hline Diagonal2 & 67.25 & 353 & 354 & 38.95 & 205 & 194 \\
\hline Diagonal1 & 423.53 & 15033 & 2001 & 315.34 & 10818 & 1448 \\
\hline Diagonal3 & 418.64 & 16311 & 2001 & 323.20 & 11385 & 1432 \\
\hline Hager & 33.78 & 626 & 176 & 58.16 & 1511 & 284 \\
\hline Generalized Tridiagonal-1 & 19.14 & 414 & 95 & 19.84 & 441 & 94 \\
\hline Extended Tridiagonal-1 & 7.98 & 35 & 32 & 6.41 & 30 & 24 \\
\hline Extended Three Exponential Terms & 2.02 & 13 & 10 & 2.48 & 32 & 12 \\
\hline Generalized Tridiagonal-2 & 28.84 & 977 & 145 & 41.16 & 1365 & 196 \\
\hline Diagonal4 & 0.42 & 9 & 4 & 1.28 & 29 & 8 \\
\hline Diagonal5 & 0.91 & 5 & 6 & 1.27 & 12 & 8 \\
\hline Extended Himmelblau & 1.70 & 19 & 10 & 4.02 & 62 & 20 \\
\hline Generalized PSC1 & 110.98 & 672 & 584 & 23.94 & 166 & 120 \\
\hline Extended PSC1 & 3.14 & 25 & 16 & 4.75 & 41 & 22 \\
\hline Extended Powel** & 1.56 & 148 & 78 & 1.02 & 111 & 50 \\
\hline Extended Block Diagonal BD1 & 2.66 & 15 & 14 & 2.91 & 30 & 16 \\
\hline Extended Maratos & 12.27 & 141 & 65 & 17.20 & 241 & 86 \\
\hline Extended Cliff & 9.16 & 71 & 44 & 4.72 & 65 & 22 \\
\hline Quadratic Diagonal Perturbed & 24.92 & 343 & 131 & 19.22 & 258 & 96 \\
\hline Extended Wood & 0.36 & 81 & 25 & 0.66 & 134 & 44 \\
\hline Scaled Quadratic SQ1 & 6.59 & 3163 & 460 & 5.67 & 3205 & 360 \\
\hline Quadratic Function QF1 & 67.39 & 2626 & 344 & 78.47 & 2949 & 378 \\
\hline Extended Quadratic Penalty QP1 & 3.36 & 40 & 19 & 8.72 & 374 & 42 \\
\hline Extended Quadratic Penalty QP2 & 6.42 & 76 & 35 & 9.84 & 133 & 50 \\
\hline A Quadratic Function QF2 & 110.25 & 5324 & 556 & 103.45 & 4822 & 492 \\
\hline Extended EP1 & 0.69 & 12 & 5 & 1.39 & 31 & 8 \\
\hline
\end{tabular}




\begin{tabular}{|c|c|c|c|c|c|c|}
\hline Extended Tridiagonal-2 & 7.16 & 72 & 39 & 8.30 & 83 & 42 \\
\hline BDQRTIC & 401.72 & 21393 & 2001 & 216.27 & 10251 & 1010 \\
\hline TRIDIA & 403.97 & 23427 & 2001 & 160.38 & 8716 & 750 \\
\hline ARWHEAD & 1.70 & 749 & 121 & 1.77 & 748 & 114 \\
\hline NONDIA (Shanno-78) & 16.36 & 310 & 86 & 44.33 & 913 & 218 \\
\hline NONDQUAR & 423.55 & 2203 & 2001 & 600.22 & 3070 & 2555 \\
\hline DQDRTIC & 2.53 & 51 & 14 & 7.42 & 199 & 38 \\
\hline Extended Rosenbrock & 7.20 & 74 & 38 & 10.33 & 153 & 52 \\
\hline EG2 & 11.64 & 486 & 59 & 5.80 & 280 & 34 \\
\hline DIXMAANA* & 0.48 & 14 & 11 & 0.73 & 27 & 16 \\
\hline DIXMAANB* & 0.67 & 19 & 16 & 0.95 & 31 & 20 \\
\hline Almost Perturbed Quadratic & 71.27 & 3120 & 361 & 75.47 & 3187 & 360 \\
\hline Tridiagonal Perturbed Quadratic & 89.06 & 3623 & 420 & 87.52 & 3468 & 392 \\
\hline DIXMAANC* & 0.66 & 21 & 16 & 0.94 & 40 & 20 \\
\hline DIXMAANE* & 8.11 & 176 & 174 & 4.98 & 109 & 102 \\
\hline Partial Perturbed Quadratic & 82.22 & 3446 & 392 & 93.06 & 3728 & 420 \\
\hline Broyden Tridiagonal & 40.69 & 1206 & 203 & 44.28 & 1303 & 208 \\
\hline total & 3544.33 & 125019 & 18279 & 2995.23 & 86211 & 14322 \\
\hline
\end{tabular}

Table 4. Statistics of the ratio.

\begin{tabular}{|c|c|c|c|c|c|c|}
\hline \multirow{2}{*}{ Problem } & \multicolumn{3}{|c|}{ DFP/HDFP } & \multicolumn{3}{|c|}{ BFGS/HBFGS } \\
\hline & Time & $f$ & $\nabla f$ & Time & $f$ & $\nabla f$ \\
\hline small 20 functions & 2.49 & 2.66 & 2.63 & 0.93 & 1.14 & 1.31 \\
\hline middle 50 functions & 1.03 & 1.29 & 1.23 & 1.58 & 2.11 & 2.09 \\
\hline large scale 50 functions & 1.23 & 1.07 & 1.27 & 1.18 & 1.45 & 1.28 \\
\hline average & 1.58 & 1.67 & 1.71 & 1.23 & 1.57 & 1.56 \\
\hline
\end{tabular}

the global convergence of this scheme and variant method using DFP and BFGS update formula. In particular, this iteration has a class of variant methods using different directions as the right-hand side vectors of (4). From our experiments, we can safely conclude that this iteration scheme improved the BFGS and DFP method on the test data sets.

\section{References}

[1] W. C. Davidon, "Variable Metric Method for Minimization,” Technical Report ANLC5990 (Revised), Argonne National Laboratory, Argonne, 1959.

[2] W. C. Davidon, "Variable Metric Method for Minimiza- tion,” SIAM Journal on Optimization, Vol. 1, No. 1, 1991, pp. 1-17. doi:10.1137/0801001

[3] R. Fletcher and M. J. D. Powell, “A Rapidly Convergent Descent Method for Minimization,” The Computer Journal, Vol. 6, No. 2, 1963, pp. 163-168.

[4] C. G. Broyden, "The Convergence of a Class of Double Rank Minimization Algorithms 2. The New Algorithms,” IMA Journal of Applied Mathematics, Vol. 6, No. 3, 1970, pp. 222-231. doi:10.1093/imamat/6.3.222

[5] R. Fletcher, "A New Approach to Variable Matric Algorithm,” The Computer Journal, Vol. 13, No. 3, 1970, pp. 317-322. doi:10.1093/comjnl/13.3.317

[6] C. G. Broyden, "The Convergence of a Class of Double Rank Minimization Algorithms 1. General Consideration,” IMA Journal of Applied Mathematics, Vol. 6, No. 1, 
1970, pp. 76-90. doi:10.1093/imamat/6.1.76

[7] D. Goldfarb, “A Family of Variable Metric Methods Derived by Variational Means,” Mathematics of Computation, Vol. 24, No. 109, 1970, pp. 23-26. doi:10.1090/S0025-5718-1970-0258249-6

[8] D. F. Shanno, "Conditioning of Quasi-Newton Methods for Function on Minimization," Mathematics of Computation, Vol. 24, No. 111, 1970, pp. 647-656. doi:10.1090/S0025-5718-1970-0274029-X

[9] K. J. Arrow, L. Hurwicz and H. Uzawa, "Studies in Linear and Nonlinear Programming," Stanford University Press, Palo Alto, 1958.

[10] F. H. Branin and S. K. Hoo, "A Method for Finding Multiple Extreme of a Function of $n$ Variables,” In: F. A. Lootsman, Ed., Numerical Method for Nonlinear Optimization, Academic Press, Cambridge, 1972.

[11] P. Q. Pan, "Differential Equation Methods for Unconstrained Optimization," Nanjing University Journal of
Computational Mathematics, in Chinese, Vol. 4, 1982, pp. 338-349.

[12] P. Q. Pan, "New ODE Methods of Equality Constrained Optimization (1): Equations,” Journal of Computational Mathematics, Vol. 10, No. 1, 1992, pp. 77-92.

[13] P. Q. Pan, "New ODE Methods for Equality Constrained Optimization (2): Algorithm,” Journal of Computational Mathematics, Vol. 10, No. 2, 1992, pp. 129-146.

[14] J. Nocedal and S. J. Wright, "Numerical Optimization,” Science Press, Beijing, 2006.

[15] J. J. More, B. S. Garbow and K. E. Hillstrome, "Testing Unconstrained Optimization Software,” ACM Transactions on Mathematical Software, Vol. 7, No. 1, 1981, pp. 17-41. doi:10.1145/355934.355936

[16] N. Andrei, "Unconstrained Optimization Test Function,” Advanced Modeling and Optimization, Vol. 10, No. 1, 2008, pp. 147-161. 